\title{
Treatment of Appendicitis With Iliopsoas Abscess by Laparoscopic One-Step Surgery
}

\author{
Kyeong Woon Choi ${ }^{\mathrm{a}}$, Woo Yong Lee ${ }^{\mathrm{a}, \mathrm{b}}$
}

\begin{abstract}
Acute appendicitis is the most common cause of acute abdomen and on rare occasions can present atypically with a retroperitoneal abscess. In this report, we present a rare case of acute appendicitis combined with iliopsoas muscle abscess. A laparoscopic appendectomy and iliopsoas abscess drainage were performed by a one-step laparoscopic procedure that did not incur any intraoperative or postoperative complications. The results of this case demonstrate that the laparoscopic approach is feasible and effective for the treatment of appendicitis complicated by retroperitoneal abscess.
\end{abstract}

Keywords: Appendicitis; Iliopsoas abscess; Retroperitoneal abscess

\section{Introduction}

Acute appendicitis is the most common surgical emergency encountered by general surgeons. In the majority of cases, the underlying cause is unknown, and on rare occasions, appendicitis can be complicated by a retroperitoneal abscess. We performed a PubMed search of published literature from 1955 to 2016 using the keywords "appendicitis", "retroperitoneal abscess", and "iliopsoas abscess", and identified 23 cases. Most cases were treated with open appendectomy with drainage, while laparoscopic surgery was performed only in three cases. In this report, we describe the successful management of complicated appendicitis with iliopsoas abscess through minimally invasive laparoscopic surgery.

\section{Case Report}

An 80-year-old woman presented to our hospital's orthope-

\section{Manuscript accepted for publication December 19, 2016}

aDepartment of Surgery, Seoul Paik Hospital Inje University College of Medicine, 9, Mareunnae-ro, Jung-gu, Seoul 100-032, South Korea

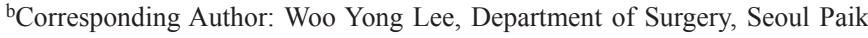
Hospital Inje University College of Medicine, 9, Mareunnae-ro, Jung-gu, Seoul 100-032, South Korea. Email: yongaaa5972@naver.com

doi: https://doi.org/10.14740/jcs313w dic clinic with a 1-month history of right hip pain. The results of a hip and pelvic radiograph were unremarkable, and there was no abdominal discomfort reported on physical examination. The patient was admitted and treated conservatively, and 3 days after admission, a computed tomography (CT) scan was performed due to uncontrollable fever, leukocytosis (up to $19,690 / \mu \mathrm{L}$ ), and right lower quadrant (RLQ) tenderness. This revealed appendicitis and a right iliopsoas abscess. The tip of the appendix was attached to the abscess without definite delineation, and enhanced peritoneal infiltration was noted around the appendix (Fig. 1A).

A decision was made to treat the patient laparoscopically. A $2 \mathrm{~cm}$ linear incision was made on the umbilicus and a glove port (Meditech Inframed, Seoul, Korea) was positioned, providing both $360^{\circ}$ wound protection and circumferential atraumatic retraction (Fig. 1B). After identification of the appendiceal tip forming fistula tract with the abscess (Fig. 2A, B), laparoscopic appendectomy and drainage were performed. In order to drain the abscess, a $5 \mathrm{~mm}$ port incision was made in the RLQ (Fig. 1B). The bacteria culture for abscess revealed Enterococcus faecalis and the patient was subsequently treated with ciprofloxacin (400 mg intravenous twice daily) and metronidazole (500 mg intravenous three times daily) for 1 week. The patient was discharged 2 weeks after surgery with a normal physical examination and normalized laboratory results. A follow-up CT revealed an interval decrease in the size of the abscess from $3.1 \times 2.6 \times 5.9$ to $1.9 \times 1.4 \times 3.9 \mathrm{~cm}$ (Fig. $2 \mathrm{C}$ ). The patient was reviewed in clinic 2 weeks after discharge, at which time she showed no signs of infection and had normal laboratory results, as well as CT evidence of complete regression (Fig. 2D).

\section{Discussion}

Appendicitis is the most common intra-abdominal condition that requires emergency surgery with a lifetime risk of $6.7 \%$ for women and $8.6 \%$ for men [1]. The majority of acute cases are managed laparoscopically; importantly, the risks and benefits of this surgical approach do not differ between complicated and uncomplicated cases. In our hospital, we have been performing laparoscopic appendectomy using the single port approach since 2011, even in cases of complicated appendicitis.

An iliopsoas abscess is a type of infectious myositis that may complicate diabetes mellitus and even minor muscle trauma [2]. Most iliopsoas abscesses are easily diagnosed via 

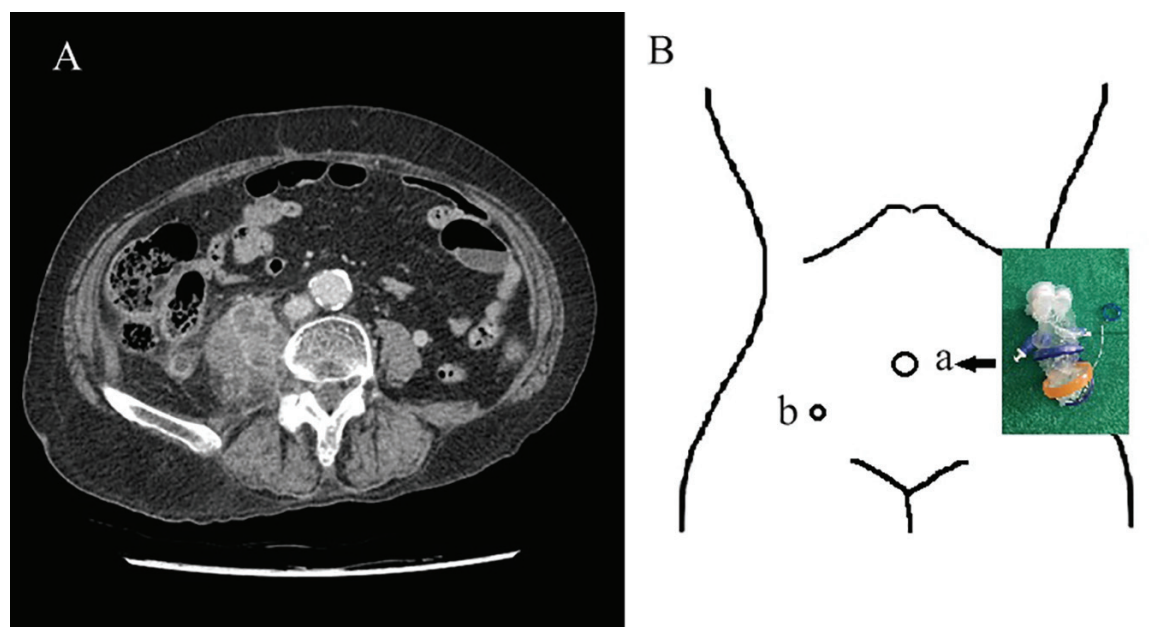

Figure 1. (A) Preoperative computed tomography (CT) image showing right multifocal iliopsoas abscesses and appendicitis. (B) Port site in operation: (a) Umbilicus, glove port (Meditech Inframed, Seoul, Korea); (b) $5 \mathrm{~mm}$ port (Laport ${ }^{\mathrm{TM}}$ Bladeless, $5 \times 75 \mathrm{~mm}$, Meditech Inframed, Seoul, Korea) for abscess drainage.

ultrasound, CT, or magnetic resonance imaging. Microbiology culture results of secondary iliopsoas abscesses are usually positive for enteric flora such as Escherichia coli, enterococci and anaerobic microorganisms, and in our case, Enterococcus faecalis was isolated [3]. Gastrointestinal inflammatory diseases such as appendicitis, Crohn's disease, diverticulitis, and colon carcinoma are the most common causes of iliopsoas abscess [4]. It was uncertain, in our case, whether the iliopsoas abscess was secondary or not, because of atypical pattern of abdominal pain and the possibility masking another bacterial culture by the fistula tract between appendix and iliopsoas abscess. CT-guided percutaneous drainage is a usually performed treatment option in iliopsoas abscess. Surgery should be considered for the failure of percutaneous catheter drainage, the presence of contraindications to percutaneous drainage and some cases of secondary abscess [5].

In this case, we successfully treated a patient with appendicitis and iliopsoas abscess through single port laparoscopic appendectomy and abscess drainage, followed by a 1 -week course of antibiotics. This surgical approach was feasible and

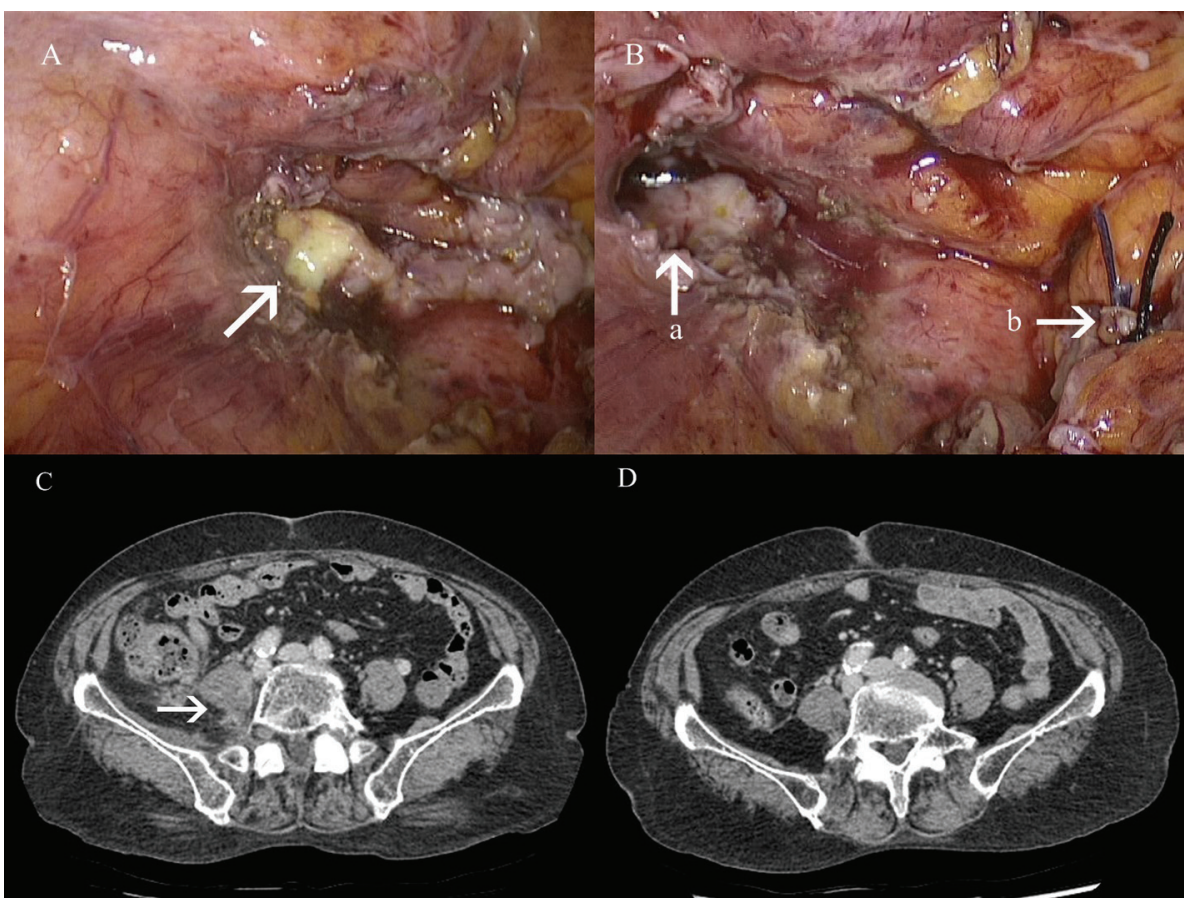

Figure 2. (A, B) Intraoperative field and (C, D) follow-up computed tomography (CT) image. (A) Appendiceal tip (arrow) with abscess, inflammatory exudate and adhesion in the right iliopsoas muscle. (B): (a) fistula tract in the right iliopsoas muscle abscess; (b) ligated appendiceal stump. (C) CT image on discharge. (D) Follow-up CT image on 2 weeks after discharge. 
there were no perioperative or postoperative complications. Advantages of the minimally invasive approach include less painful recovery, fewer postoperative complications, and improved cosmetic results. For these reasons, laparoscopy should be the preferred alternative to open surgery in cases of complicated appendicitis with retroperitoneal iliopsoas abscess.

\section{Conclusion}

This is the case report of one-step minimal invasive laparoscopic treatment for coexisting iliopsoas abscess and appendicitis. We hope this paper will help in treatment in similar patients.

\section{References}

1. Addiss DG, Shaffer N, Fowler BS, Tauxe RV. The epide- miology of appendicitis and appendectomy in the United States. Am J Epidemiol. 1990;132(5):910-925.

2. van den Berge $M$, de Marie S, Kuipers T, Jansz AR, Bravenboer B. Psoas abscess: report of a series and review of the literature. Neth J Med. 2005;63(10):413-416.

3. Charalampopoulos A, Macheras A, Charalabopoulos A, Fotiadis C, Charalabopoulos K. Iliopsoas abscesses: diagnostic, aetiologic and therapeutic approach in five patients with a literature review. Scand J Gastroenterol. 2009;44(5):594-599.

4. Huang JJ, Ruaan MK, Lan RR, Wang MC. Acute pyogenic iliopsoas abscess in Taiwan: clinical features, diagnosis, treatments and outcome. J Infect. 2000;40(3):248255.

5. Cantasdemir M, Kara B, Cebi D, Selcuk ND, Numan F. Computed tomography-guided percutaneous catheter drainage of primary and secondary iliopsoas abscesses. Clin Radiol. 2003;58(10):811-815. 